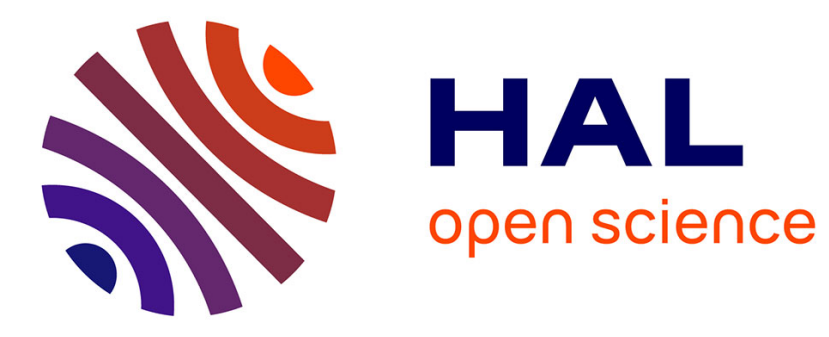

\title{
Allowing People to Communicate After a Disaster Using FANETs
}

\author{
Frédéric Guinand, François Guérin, Pawel Łubniewski
}

\section{To cite this version:}

Frédéric Guinand, François Guérin, Pawel Łubniewski. Allowing People to Communicate After a Disaster Using FANETs. Nets4Cars/Nets4Trains/Nets4Aircraft 2020: Communication Technologies for Vehicles, pp.181-193, 2020, 10.1007/978-3-030-66030-7_16 . hal-03352602

\section{HAL Id: hal-03352602 \\ https://hal.science/hal-03352602}

Submitted on 23 Sep 2021

HAL is a multi-disciplinary open access archive for the deposit and dissemination of scientific research documents, whether they are published or not. The documents may come from teaching and research institutions in France or abroad, or from public or private research centers.
L'archive ouverte pluridisciplinaire HAL, est destinée au dépôt et à la diffusion de documents scientifiques de niveau recherche, publiés ou non, émanant des établissements d'enseignement et de recherche français ou étrangers, des laboratoires publics ou privés. 


\title{
Allowing People to Communicate after a Disaster using FANETs ${ }^{\star}$
}

\author{
Frédéric Guinand ${ }^{1,2[0000-0002-7915-2781]}$, François Guérin ${ }^{3[0000-0003-4163-4459]}$, \\ and Pawel Łubniewski2 [0000-0003-2446-7644] \\ 1 Normandy Univ, UNIHAVRE, LITIS Lab, France \\ frederic.guinand@univ-lehavre.fr \\ 2 Cardinal Stefan Wyszynski University in Warsaw, Poland \\ \{f.guinand,p.lubniewski\}@uksw.edu.pl \\ 3 Normandy Univ, UNIHAVRE, GREAH Lab, France \\ francois.guerin@univ-lehavre.fr
}

\begin{abstract}
When a disaster occurs, during a long period of time people suffer to have no means to communicate with their relatives. The presented work aims at proposing a solution composed of a ground station located nearby the damaged area coupled with a swarm of drones. The ground station plays the role of a gateway between cellular networks, that are still up but out of reach of people, with drones that carry messages from and to people located in the disastered region. We analyze the possibility of deploying drones with fixed positions and a more flexible solution allowing drones to move but at the cost of intermittent communications, allowing only sms-like messages. We show that using the same number of drones, allowing drones to move improves dramatically the coverage of people with respect to a FANET in which drones stay at a fixed position. We also show that even for a very restricted number of drones, for reasonnable communication ranges, almost all the people benefit from an important average connected time.
\end{abstract}

Keywords: Flying Ad Hoc Networks · Disaster · Intermittent Communication Network · Coverage · Swarm of Drones · UAV

\section{Introduction}

In the two last reports of IPCC ${ }^{4}$ many clues show that Global Warming entails meteorological events more and more sudden and strong like hurricanes, forest fires and floods. Most often, communication networks become out of order during periods ranging from few days to several weeks. This is especially true when the events are floods (Hurricane Katrina in 2005 for instance or very recently in South of France (Vésubie and Roya Valleys)) because the accesses are cut off for a long period of time. The deployment of equipments for restoring cellphones networks usually requires from several days up to weeks since electricity is often

\footnotetext{
* Supported by SolarFarm Project

${ }^{4}$ Intergovernmental Panel on Climate Change
} 
missing as well as safe infrastructures (roads) for moving to the right places and because first aid teams have logically the priority for accessing the damaged zones.

If the conditions during such meteorological events are not favorable to the deployment of medium sizes drones, usually after some hours such machines can safely fly in the sky above the concerned areas. In addition, after a first overview of the region, the damaged zones are usually identified and geographically bounded (GPS points for instance).

In most works dealing with communication networks recovery using drones, the primary goal of the temporary network is to support rescue teams. However, as reported by testimonies of people living these catastrophes, communicating as soon as possible with their relatives is one of their priority. One possibility for offering people living in these areas a way of communicating with people living outside consists in building a temporary and infrastructureless network able to relay messages between these two populations. The connectivity constraint of such a network may be relaxed since the communication between people located inside and outside the damaged area can be intermittent (like sms). Moreover, as energy consumption for hovering or for moving have same magnitude, thus moving drones scenarios can be considered as alternative solutions to a full-time connected network.

The network considered in this work is composed of a ground station, acting as a gateway with cellular phone networks still up, and drones able to communicate with each other, with the gateway and with people. As a first attempt to address this problem we consider that drones are randomly distributed over the area. The main goal of this preliminary work is to determine the impact of some swarm's characteristics on messages delivery and area coverage. The number of uavs, communication range (people-drone and drone-drone) and drones' moving behavior are discussed and simulations results are analyzed.

\section{Related Works}

The problem of communication networks recovery in case of disaster have received much attention these last decades [6,3], and many works have also been dedicated to the possibility of building ad hoc networks using drones [1].

In order to obtain a clear view of the disaster area, [5] propose to build a network of camera embedded by drones. The goal of the work consists in computing the best position of the drones such as to maintain the connectivity of the communication network while ensuring a correct coverage of the area. For solving the problem the author model it as an ILP problem for which a solution is computed offline. Unlike this centralized approach, Maza and his colleagues propose a real-time solution for deploying UAVs and sensors for disaster management. However, the focus is mainly on task allocation and cooperation bertween drones rather than communications problems that are not explicitely taken into account [4]. More recently, in [7] the proposed solution brings into play two types of drones. Rescue drones, performing tasks directly related to the actions of the 
rescue teams, and relay drones for maintaining the connectivity between rescue drones and a gateway located outside of the damaged area. The problem addressed is the minimization of the number of relay drones for guaranteeing the connectivity of the network composed by the gateway and the rescue drones.

\section{Problem, Model and Algorithm}

The problem can be expressed as the deployment of a swarm of drones within an area defined by a set of GPS positions. Without loss of generality we consider a square area of dimensions $L \times L$ with the gateway located at one corner.

The swarm has to self-organize such that messages can be sent from peopleto-drone and routed from drone-to-drone and from drone-to-gateway to be delivered outside of the area. Conversely, incoming messages should be routed from the gateway to people. Technical issues at the level of the gateway are out of the scope of this work and will not be discussed further as well as technologies used for drone-to-drone and drone-to-gateway communications. We only assume that drone-to-drone and drone-to-gateway communication range is equal to $r_{d 2 d}$. It is supposed that each drone can serve as an access point to which people can connect to. Communication range to and from these access points is equal to $r_{d 2 p}$. People are supposed to be uniformly and randomly distributed over the area and their number is equal to $m=\delta \times L \times L$ where $\delta$ is the density of people in the considered region.

The general algorithm executed by every drone is reported in Algorithm 1. In a first phase, each drone starts by an initialization phase: get the limits of the area, its initial position, a destination in the area, and a set of waypoints or a shape defining the mobility pattern. Note that apart from the limits of the area, the destination can be randomly chosen as well as the waypoints or the mobility pattern. It then takes off and moves toward this point. Once arrived, it starts the access point. The UAV then iterates on three tasks: (i) accept connections from people (ii) manage messages and (iii) move according to the chosen mobility pattern and get its current position while moving.

Messages are managed by different tasks processed simultaneously every drone $D$ :

1. reception of messages sent by people connected to $D$,

2. emission of a message containing $D$ 's position to neighbors drones,

3. reception of people's messages routed by neighbor drones

4. routing of people's messages to neighbors closer to the gateway than $D$, or directly to the gateway if such a connection exists.

When a drone reaches its position and starts an access point. People in the communication range of this drone establish a connection with the access point. Messages are then emitted from them to the drone. In the same time, using another communication channel, drones emit some messages containing their initial position (dest) to their neighbors. Note that, since drones are moving, the neighborhood of each of them may change all the time. During its movement, a 


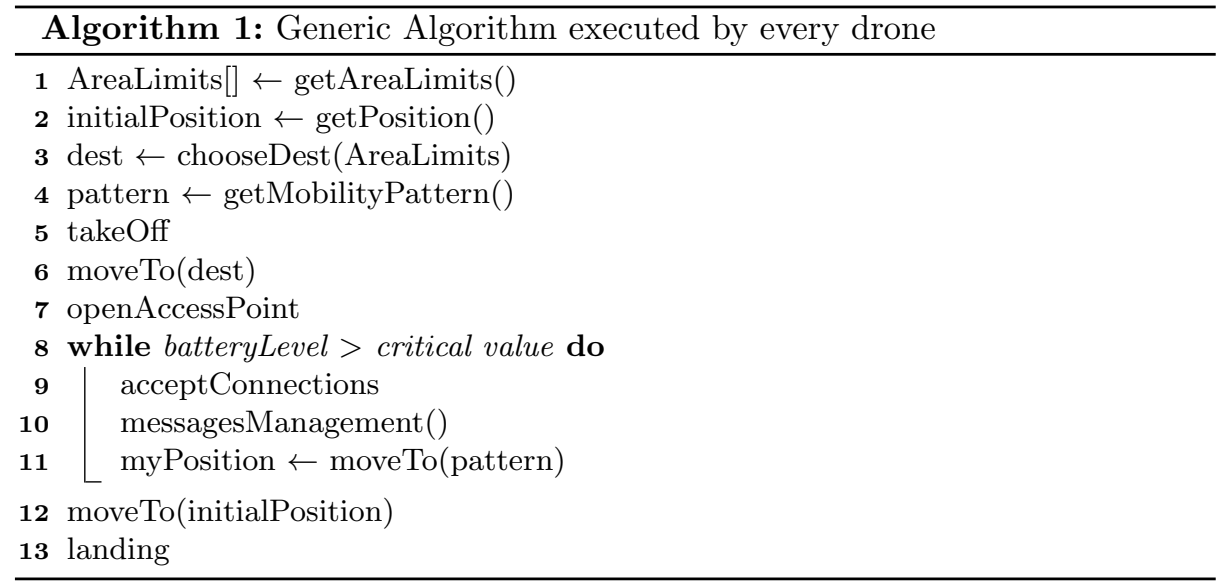

drone can route people's messages to another drone if the initial position of this latter is closer to the gateway. Finally, when directly connected to the gateway, a drone delivers all the carried messages.

Remark: we only consider messages going from people to the gateway and not messages coming from the gateway to people. Note however that messages from a person to the gateway can be stamped by both the phone number of the person and the GPS position of the drone to which the person was connected. Thus, when a message, coming from outside, in destination to a given phone number arrives to the gateway, it recover the GPS position corresponding to the phone number and the mechanism used for outgoing messages can be applied for routing the message to the intended person.

We study first, in Section 4.1, the likelihood for the network, formed by the gateway and drones, to be connected, as well as the percentage of people located in the communication range of drones in the case where drones remain at a static position in the air.

In a second part, we consider our solution, defined by two parameters: the number of drones and the mobility pattern. For a set of fixed values of $\delta, L, r_{d 2 d}$ and $r_{d 2 p}$ we measure the percentage of people connected to the drones and the average delay for their messages to reach the gateway.

\section{Simulations and Analysis}

In this section we investigate the impact of the mobility on the coverage of people by drones. All simulation were performed using GraphStream ${ }^{5}$, a dynamic graphs library [2].

\footnotetext{
${ }_{5}^{5}$ graphstream-project.org
} 


\subsection{Without Mobility}

First remark that if drones remain at fixed positions, in order to fullfil the requirements (routing messages from people to the gateway), the drones network (including the gateway) has to be connected and all the people have to be connected to a drone. As illustrated by Figure 1 covering all the people is a big challenge if uavs are randomly distributed over the area.

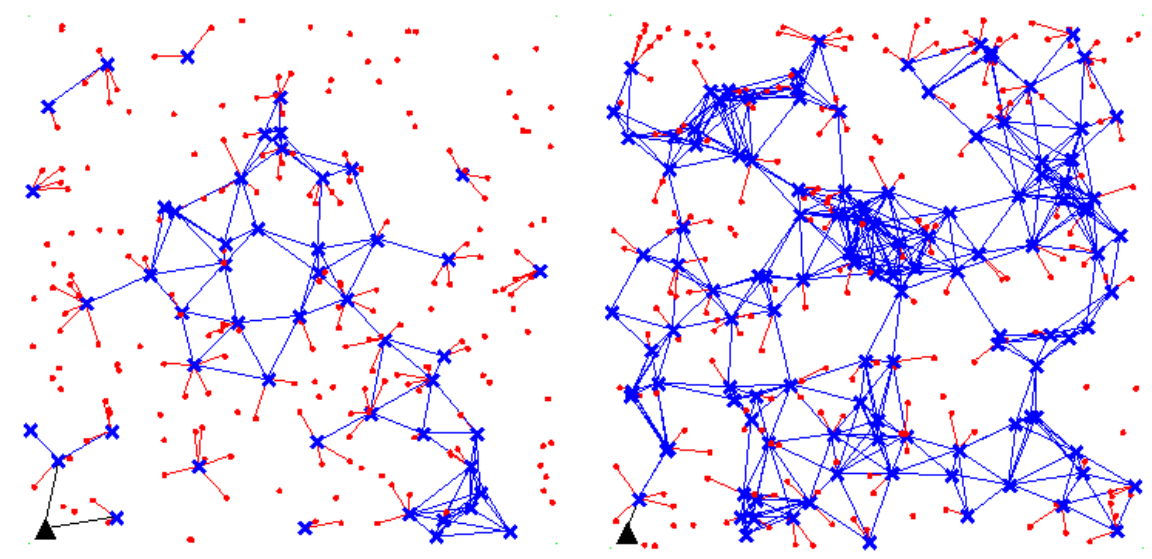

Fig. 1. Two networks obtained for fixed drones' positions, randomly chosen. Parameter values: $r_{p 2 d}=80, L=1000$ and $r_{2 d 2}=150$. On the left side $n=50$ and $n=120$ on the right side. Crosses are drones, points are people, the antenna is represented by a black triangle at the bottom left corner of the area.

If uavs are randomly distributed over the area, if $S=\frac{\pi r^{2}}{L^{2}}$ denotes the surface covered by one drone (with $r$ the communication range and $L^{2}$ the surface of the area), then for $n$ drones, the probability of the network to be connected is approximately $e^{-\alpha}$ where $\alpha=n \times e^{n \times S}$

From Figure 2 we can argue that if drones are randomly distributed over the area and if the communication range of the drones is approximately one fifth of the size of the damage area, about 200 drones are needed by square kilometer for ensuring the connectivity.

If drones are not randomly distributed over the area (supposed to be a square), if the communication range is $r$ and the length of one side is $L$, then the minimum number of drones needed for covering the area while maintaining the connectivity of the network is equal to: $\left(\frac{L}{r}+1\right)^{2}$. 


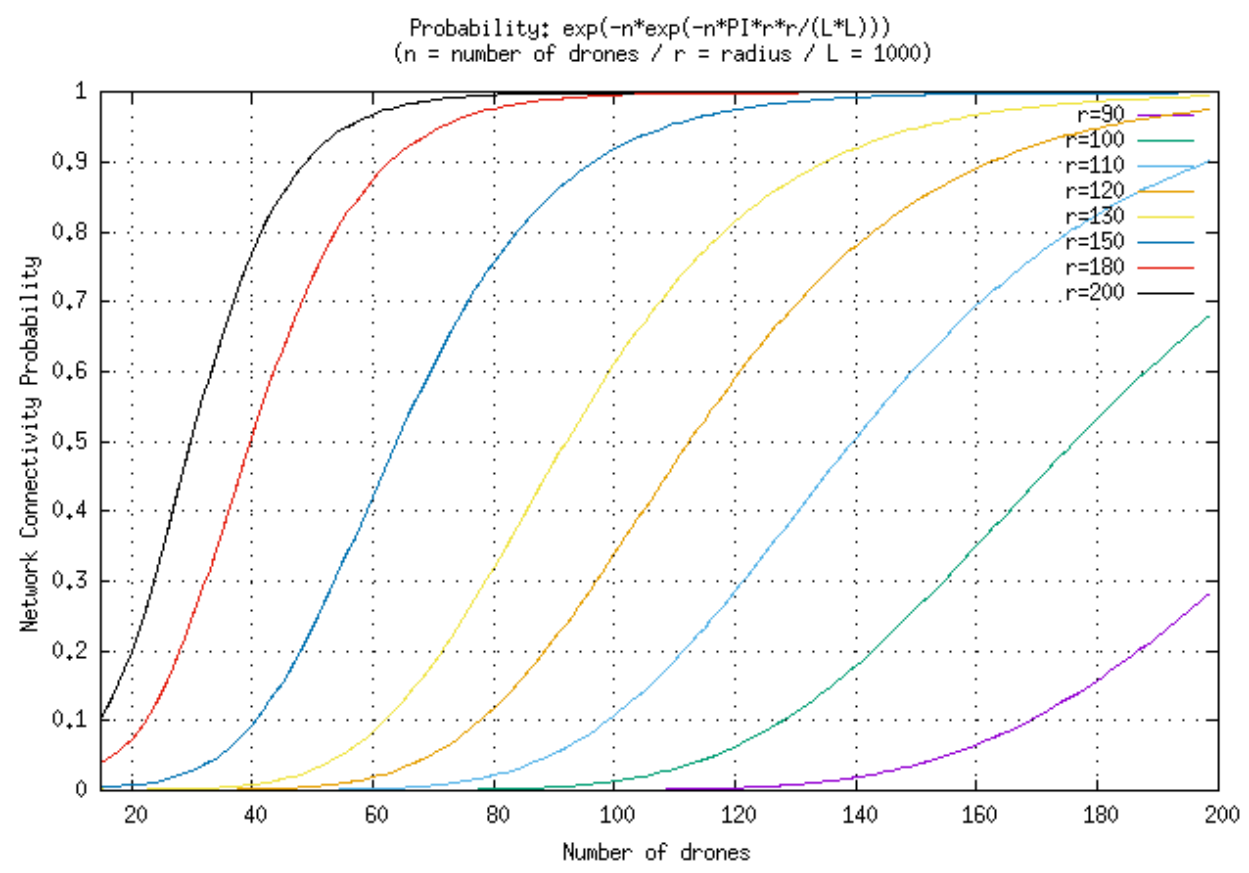

Fig. 2. Network Connectivity Probability according to the number of drones and their communication range.

Thus for an area of $4 \mathrm{~km}^{2}$ if the communication range of drones are around 200 meters, the number of drones required for that task is 120. Figure 3 represents this minimum number for various values of $r$, the communication range, and $L$ the size of the area.

In order to reduce the number of drones necessary for answering the initial question, our approach consists in allowing drones to move around their initial position such as to cover a larger area. The direct consequence of this choice is that anytime connectivity can no longer be guaranteed. In the context of this study where only sms-like messages are routed within the network this choice does not prevent message delivery but may entail an additional delay. The next sections attempt to measure the impact of the number of drones and of the mobility on the coverage of the people, the average people to drone connected time and on the delay of messages delivery.

\subsection{With Mobility}

The mobility pattern considered in this section is a circle. Each drone randomly chooses the center of a virtual circle located at a distance between $r_{d 2 d} / 2$ and 


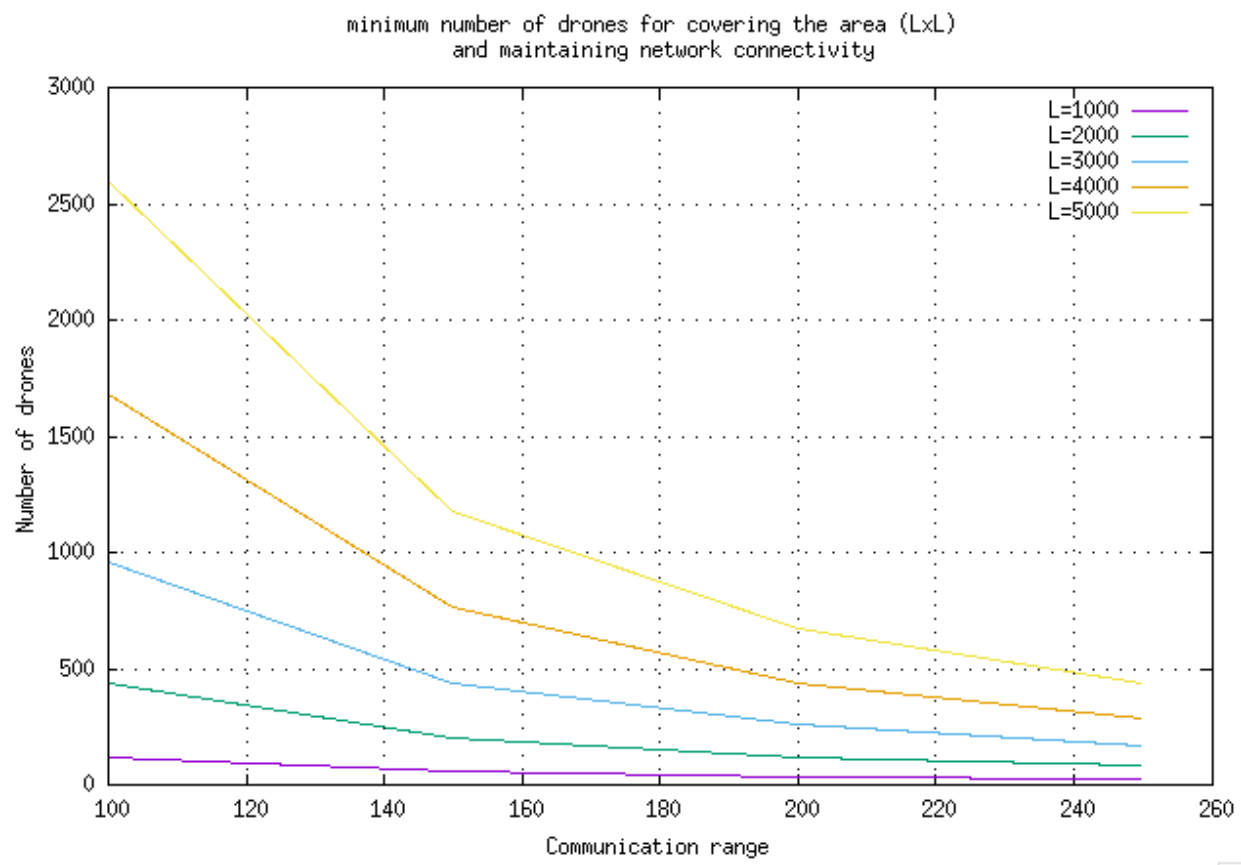

Fig. 3. Minimum number of drones required for maintaining network connectivity and guaranteeing the coverage of the area.

$r_{d 2 d}$. It may happen that the center of the virtual center is located outside of the area of interest. It then moves along this circle while its battery level is greater than a given critical level. Each person can only connect to one drone at a time. For the simulations we set some parameters: the number of people is set to 200 , the area is a square of size $1000 \times 1000$ and we set the drone-to-drone communication range to $r_{d 2 d}=150$. We study the impact of the number of drones and of the people-to-drone communication range $\left(r_{p 2 d}\right)$ on:

- the percentage of people covered by the swarm

- the percentage of time during which people are connected to drones

- the number of messages that successfully reach the gateway

- the average delay for a message to reach the gateway

Note that: (i) each person emits only one message, (ii) no message is lost and (iii) during one time step, a message can cross more than one link between drones.

Each point of the figures in the following sections and paragraphs corresponds to a set of fixed parameters and results from 100 runs.

\subsection{Coverage}

The coverage does not depend on drone-to-drone communication range, but only on the number of drones, on the distribution of people in the area and on 
the people-to-drone communication range. Each run is performed with another random uniform distribution of people in the area. On Figure 4 simulation results are reported.

The addition of the mobility dramatically decreases the number of required drones for ensuring the connectivity of people with respect to a scenario for which drones hover over a fixed position. If people-to-drone communication range is equal to 150 then as few as 20 drones are enough for covering more than $95 \%$ of people. This number should be compared when the drones of the swarm remain at fixed positions in the air. In order to obtain the same coverage value, keeping the same $r_{p 2 d}=80$ value, at least 200 drones have to be deployed in the swarm. Note also that with 10 drones and a communication range limited to 50 more than $50 \%$ of people are reached.

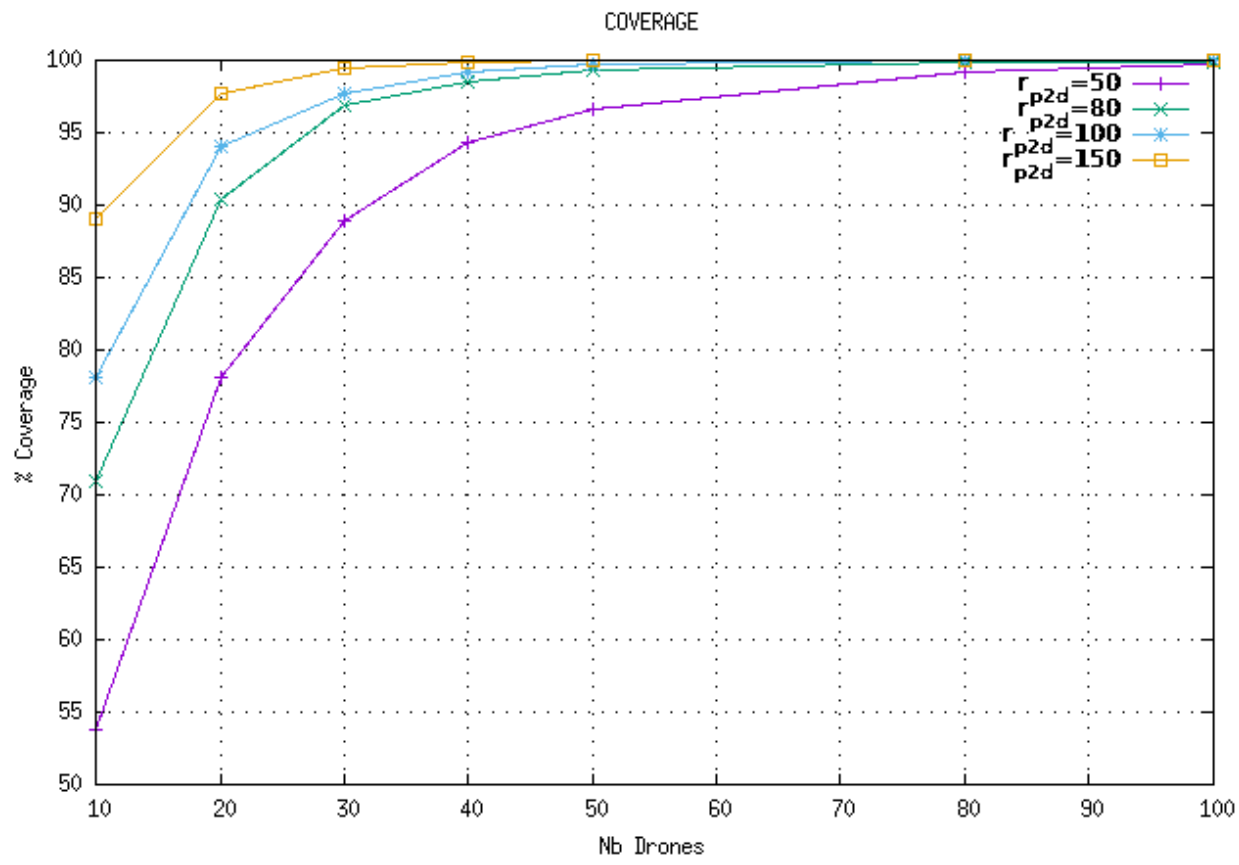

Fig. 4. Percentage of people covered by the swarm according to the number of drones ( $\mathrm{x}$ axis) and to people-to-drone communication range.

\subsection{Connection Time}

The second measure focuses on the average people-to-drone connected time. If the solution without mobility ensure a constant connection between any con- 
nected person to the network, it is no longer guaranteed when drones are moving. However the average time during which each person can be connected to one drone increases up to more than $80 \%$ when using 40 drones with $r_{p 2 d}=150$. In comparison with the static scenario (drones hover over a fixed position), using five times less drones (40 instead of 200), can achieve a QoS up to 4/5 of the optimum (permanent connection). In addition, as the mobility pattern is a circle, connections are periodically re-established.

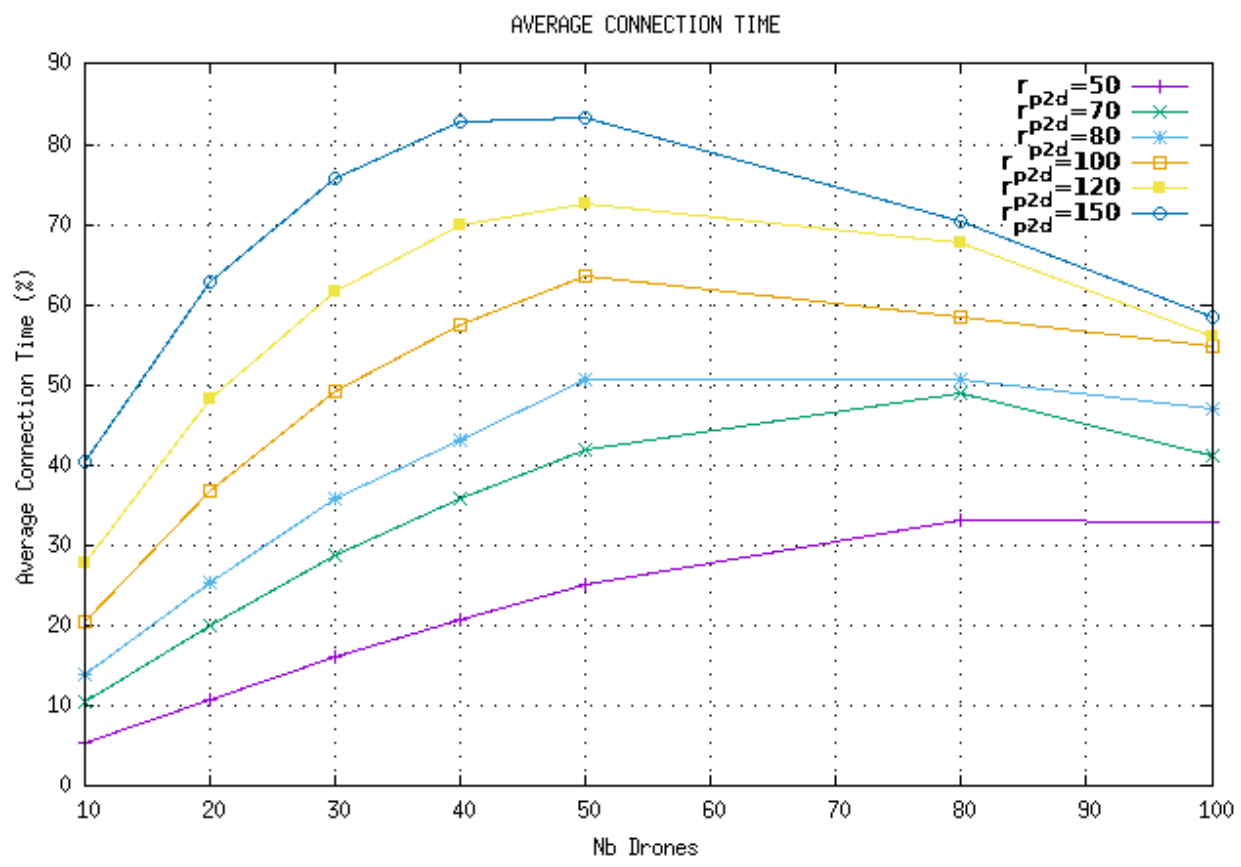

Fig. 5. Percentage of time during which people are connected to drones, according to the number of drones (x axis) and to people-to-drone communication range.

One point is currently not well understood, the average connection time seems to decrease when the number of drones increases. We plot in Figure 6 the variation of the average connection time for the 100 runs with respect to the number of drones when $r_{p 2 d}=150$ (distributions are sorted). The result is quite surprising and we are currently working for explaining this phenomenon. 


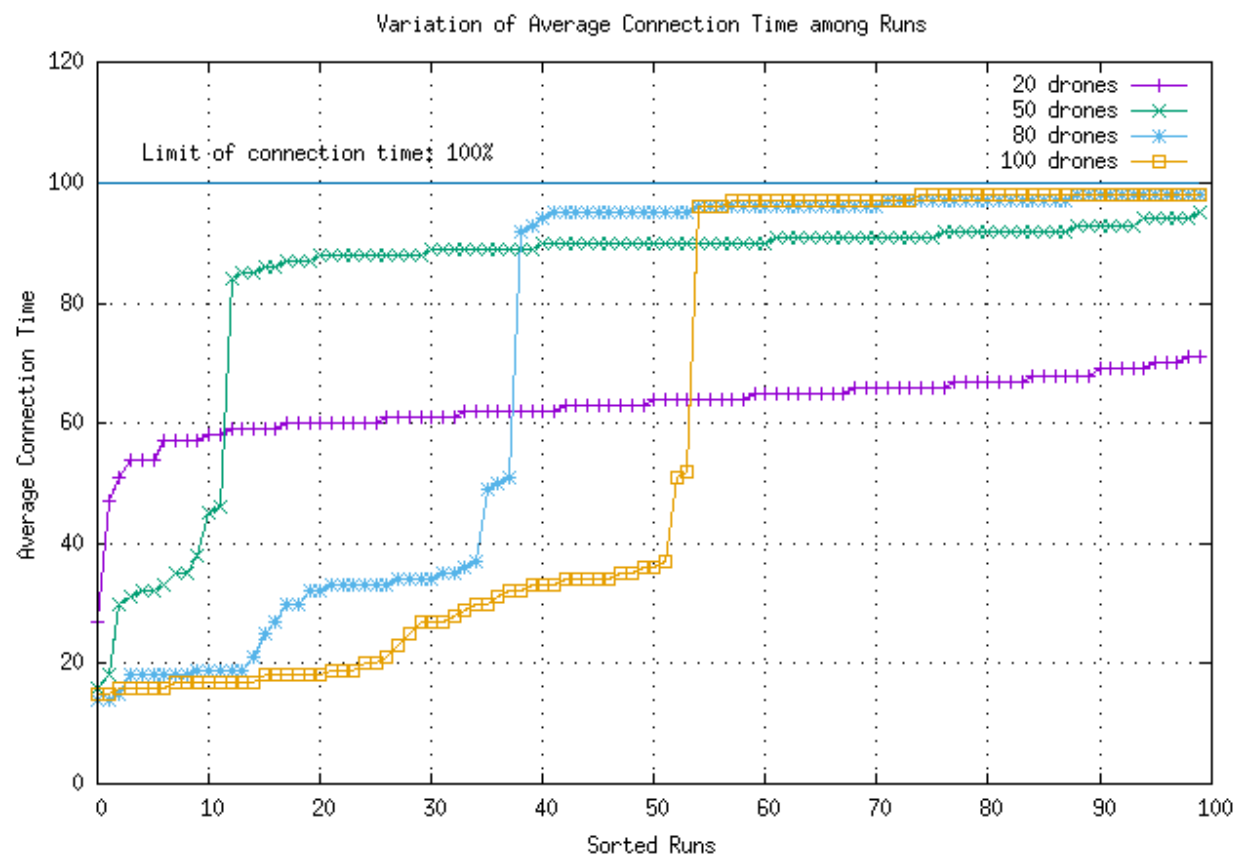

Fig. 6. Distribution of the Average Connection Time for the 100 runs performed for different number of drones and for $r_{p 2 d}=150$.

\subsection{Delivered Messages and Delay}

Message delivery is no always successful, it may happen that the gateway is never connected to any drone. The table below reports the number of times this situation occurs depending on the number of drones and on people-to-drone communication range.

\begin{tabular}{|c|c|c|c|c|c|c|c|c|}
\hline \#drones $/ r_{p 2 d}$ & 50 & 70 & 80 & 90 & 100 & 120 & 150 \\
\hline \hline 10 & 44 & 43 & 36 & 33 & 36 & 38 & 34 \\
20 & 16 & 18 & 9 & 18 & 13 & 17 & 11 \\
30 & 5 & 4 & 3 & 6 & 4 & 6 & 11 \\
40 & 1 & 3 & 3 & 1 & 1 & 2 & 7 \\
50 & 0 & 0 & 2 & 0 & 1 & 1 & 1 \\
80 & 0 & 0 & 0 & 0 & 0 & 0 & 0 \\
100 & 0 & 0 & 0 & 0 & 0 & 0 & 0 \\
\hline
\end{tabular}

When the gateway is intermittently connected to some drones, some messages are delivered. Figure 7 represents the average number of delivered messages according to the number of drones and people-to-drone communication range. As illustrated by the graphics, the impact of $r_{p 2 d}$ is negligible, only the number of drones matters. Additional experiments should be performed in order to measure 
the impact of $r_{d 2 d}$ on the same metrics. These results suggest that even if almost all people are connected ( 20 drones and $r_{p 2 d}=150$ ), the network is not connected and there exist probably several connected components that never connect with each other, a kind of archipelago of isolated groups of connected people.

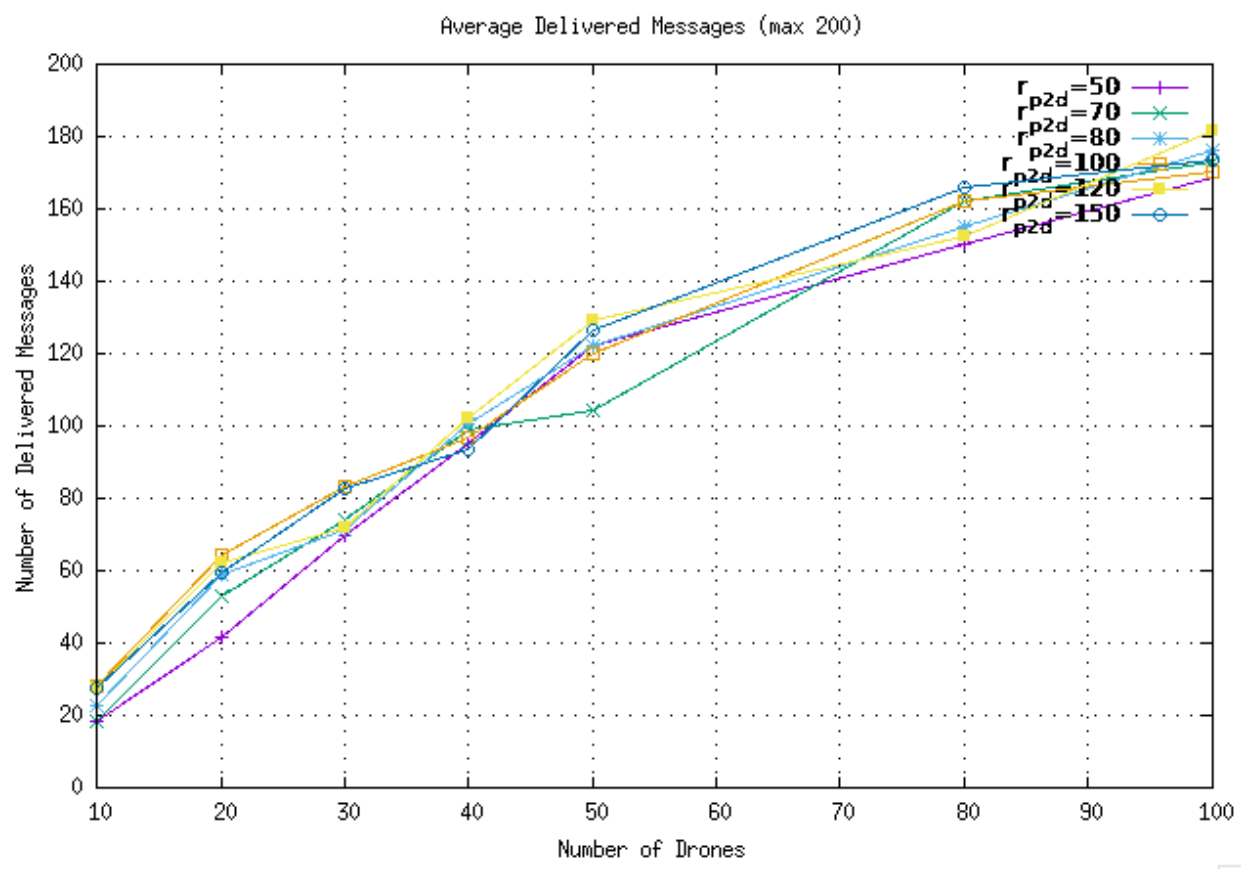

Fig. 7. Number of delivered messages with respect to the number of drones and peopleto-drone communication range.

The last proposed measure concerns the delay for routing messages from the source (people) to the gateway. The form of the curve can be explained by relying on connectivity. When the network is poorly connected, even along time, only the messages coming from the people and thus drones which positions are close to the gateway are delivered. For such messages, the delay is short. When the connectivity is better at the network level, more messages are delivered, coming from drones located farther from the gateway, entailing an increase in the delay. But the intermittent connectivity between groups entails also some delays which are partially removed when the number of drones increases. This last point explains the decrease of the value of the average delay when the number of drones increases. 


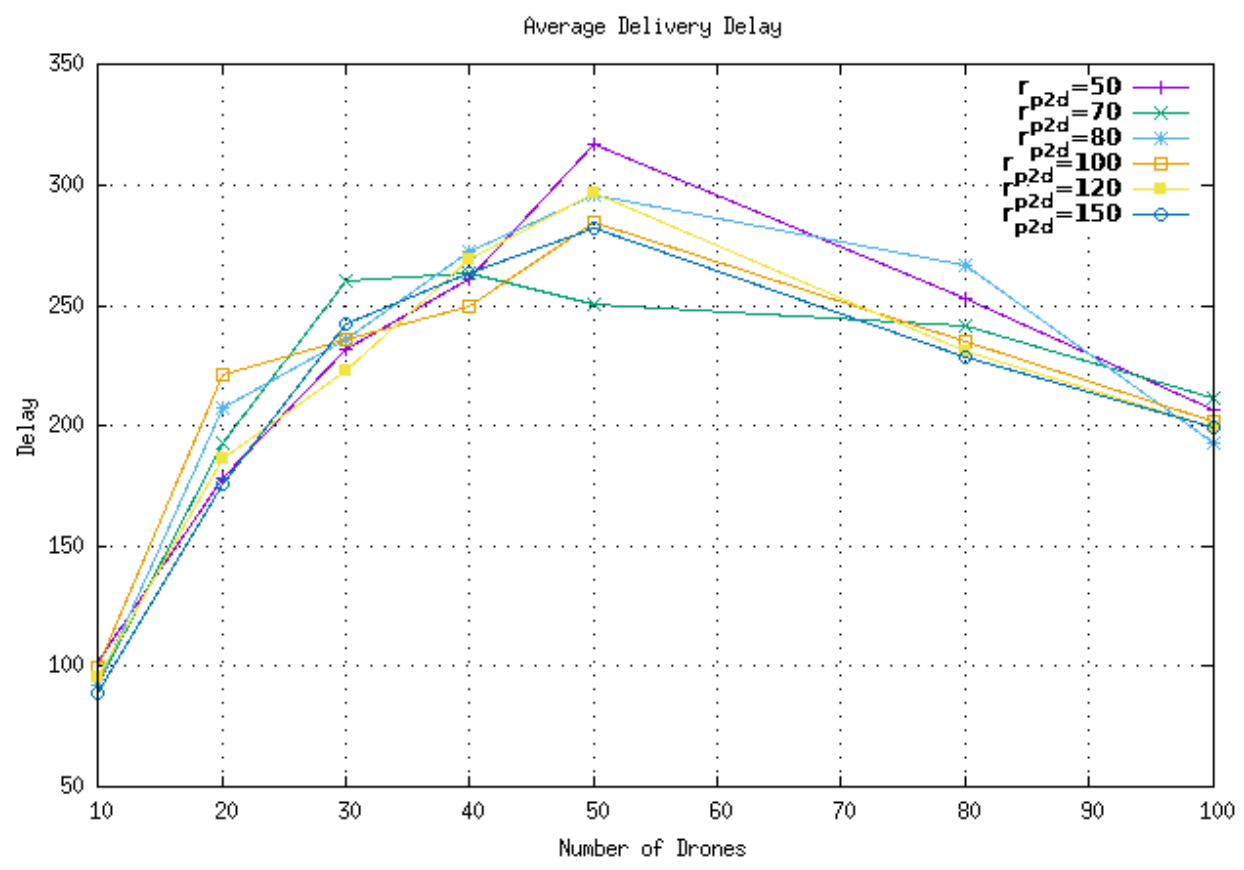

Fig. 8. Average delivery delay of messages.

\section{Conclusion and Perspectives}

When a disaster occurs in some regions with limited access, like mountains, deep valleys, or poorly connected areas, the deployment of a temporary communication network composed of a ground station and a swarm of drones may be a relevant solution for offering people a way of communicating with their relatives. However, covering the whole damaged area may require a large number of drones. We have shown that if we allow drones to move over this area instead of hovering over a fixed position, the required UAVs number is much smaller. But this economy comes at the cost of intermittent communications, preventing people to use real time streaming applications.

The solution envisioned relies on a randomly distribution of drones over the area. While this distribution is not optimized it gives some ideas for covering an unknown area with a restricted number of UAVs. One weakness of such a distribution is that in some cases, the gateway is never connected to any drone and thus no message is delivered.

The solution may be improved in many ways and deserves further investigations. The first point that could be improved is the choice of a random location in the area as the initial drones' positions. We may envisioned a new version in which drones are communicating with each other in order to avoid closeness or too large distances. But this can only be done during the first phase of the 
deployment, not studied in this work, when drones are moving from the ground station to their assigned position, since their number is too small for ensuring the connectivity of the network once in place.

The second point refers to the balance between the number of drones and the average people-to-drone connected time or the percentage of connected people (for a given value of $r_{p 2 d}$ ). This problem deserves a further study when the mobility pattern is a parameter. Some questions can be raised about the performances of different mobility patterns, in terms of average connected time, elapsed time between two connections, percentage of people connected, or message delivery delays.

\section{References}

1. Bekmezci, İ., Sahingoz, O.K., Temel, Ş.: Flying ad-hoc networks (FANETs): A survey. Ad Hoc Networks 11(3), 1254-1270 (May 2013). https://doi.org/10.1016/j.adhoc.2012.12.004, https://doi.org/10.1016/j. adhoc. 2012.12.004

2. Dutot, A., Guinand, F., Olivier, D., Pigné, Y.: Graphstream: A tool for bridging the gap between complex systems and dynamic graphs. In: Alaoui, A., Bertelle, C. (eds.) Proceedings of Emergent Properties in Natural and Artificial Complex Systems. Satellite Conference within the 4th European Conference on Complex Systems (ECCS'2007), October 4-5. Dresden, Germany. pp. 63-72 (2007)

3. Franzolini, J., Guinand, F., Olivier, D.: Mobile ad hoc network designed to communicate during crisis. In: Report EUR 26719 EN - Proceedings of the 43rd ESReDA seminar: land use planning and Risk-informed decision making. Oct. 22-23. Rouen (France). pp. 387-402 (2014)

4. Maza, I., Caballero, F., Capitán, J., de Dios, J.R.M., Ollero, A.: Experimental results in multi-UAV coordination for disaster management and civil security applications. Journal of Intelligent \& Robotic Systems 61(1-4), 563585 (Dec 2010). https://doi.org/10.1007/s10846-010-9497-5, https://doi.org/10. $1007 / \mathrm{s} 10846-010-9497-5$

5. Quaritsch, M., Kruggl, K., Wischounig-Strucl, D., Bhattacharya, S., Shah, M., Rinner, B.: Networked UAVs as aerial sensor network for disaster management applications. e \& i Elektrotechnik und Informationstechnik 127(3), 5663 (Mar 2010). https://doi.org/10.1007/s00502-010-0717-2, https://doi.org/10. 1007/s00502-010-0717-2

6. Reina, D.G., Askalani, M., Toral, S.L., Barrero, F., Asimakopoulou, E., Bessis, N.: A survey on multihop ad hoc networks for disaster response scenarios. International Journal of Distributed Sensor Networks 2015, 1-16 (2015). https://doi.org/10.1155/2015/647037, https : //doi .org/10.1155/2015/647037

7. Yang, T., Foh, C.H., Heliot, F., Leow, C.Y., Chatzimisios, P.: Self-organization drone-based unmanned aerial vehicles (UAV) networks. In: ICC 2019 2019 IEEE International Conference on Communications (ICC). IEEE (May 2019). https://doi.org/10.1109/icc.2019.8761876, https://doi.org/10.1109/icc. 2019.8761876 